University of Wollongong

Research Online

Faculty of Science, Medicine and Health -

Papers: part A

Faculty of Science, Medicine and Health

$1-1-2010$

\title{
Using Generalised Dissimilarity Models and many small samples to improve the efficiency of regional and landscape scale invertebrate
} sampling

Michael B. Ashcroft

Australian Museum, ashcroft@uow.edu.au

John R. Gollan

Australian Museum

Daniel P. Faith

Australian Museum

Gareth A. Carter

Australian Museum

Scott A. Lassau

Australian Museum

See next page for additional authors

Follow this and additional works at: https://ro.uow.edu.au/smhpapers

Part of the Medicine and Health Sciences Commons, and the Social and Behavioral Sciences

Commons

\section{Recommended Citation}

Ashcroft, Michael B.; Gollan, John R.; Faith, Daniel P.; Carter, Gareth A.; Lassau, Scott A.; Ginn, Scott G.; Bulbert, Matthew W.; and Cassis, Gerasimos, "Using Generalised Dissimilarity Models and many small samples to improve the efficiency of regional and landscape scale invertebrate sampling" (2010). Faculty of Science, Medicine and Health - Papers: part A. 3106.

https://ro.uow.edu.au/smhpapers/3106

Research Online is the open access institutional repository for the University of Wollongong. For further information contact the UOW Library: research-pubs@uow.edu.au 


\title{
Using Generalised Dissimilarity Models and many small samples to improve the efficiency of regional and landscape scale invertebrate sampling
}

\author{
Abstract \\ It is rarely cost-effective to survey invertebrates for use in systematic conservation planning activities. \\ The efficiency of sampling methods needs to be improved, and this is especially important at landscape \\ and regional scales. We investigated two methods that could be used to improve regional scale sampling \\ efficiency using a case study of ants, beetles, flies, bugs, spiders and wasps from the semi-arid Pilbara \\ region of Western Australia. First, Generalised Dissimilarity Models (GDMs) were used to divide the region \\ into landscapes with relatively homogeneous communities and environmental conditions. We found that \\ some of these landscapes were large, and a low sampling density could be employed in these areas due \\ to the low spatial turnover in species. Other landscapes were 1-2 orders of magnitude smaller, and a \\ higher sampling density should be employed to capture the high species turnover and unique species in \\ these areas. Variation of sampling density based on landscape dimensions could vastly improve survey \\ efficiency. Second, we investigated whether one large sample or five small samples were a more efficient \\ method to estimate the species composition of each landscape. We found that five small samples \\ captured a higher proportion of landscape scale species richness for a fixed sampling effort, and was \\ therefore a more efficient method to determine the species composition of the landscape. Combining five \\ small samples also resulted in less sample variability than one large sample, which increases statistical \\ power to detect changes. We concluded that GDM was an effective method to increase sampling \\ efficiency, because it allowed sampling density to vary according to the spatial turnover in species. Using \\ many small samples is a more efficient method to capture the species composition of landscapes than a \\ single large sample with an equivalent sample size.

\section{Disciplines} \\ Medicine and Health Sciences | Social and Behavioral Sciences

\section{Publication Details} \\ Ashcroft, M. B., Gollan, J. R., Faith, D. P., Carter, G. A., Lassau, S. A., Ginn, S. G., Bulbert, M. W. \& Cassis, G. \\ (2010). Using Generalised Dissimilarity Models and many small samples to improve the efficiency of \\ regional and landscape scale invertebrate sampling. Ecological Informatics, 5 (2), 124-132.
}

\section{Authors}

Michael B. Ashcroft, John R. Gollan, Daniel P. Faith, Gareth A. Carter, Scott A. Lassau, Scott G. Ginn, Matthew W. Bulbert, and Gerasimos Cassis 
Using Generalised Dissimilarity Models and many small samples to improve the efficiency of regional and landscape scale invertebrate sampling

Michael B. Ashcroft ${ }^{*}$

John R. Gollan

Daniel P. Faith

Gareth A. Carter ${ }^{1}$

Scott A. Lassau ${ }^{2}$

Scott G. Ginn

Matthew W. Bulbert ${ }^{3}$

Gerasimos Cassis ${ }^{4}$

Institution for all authors: Australian Museum, 6 College St, Sydney, NSW, 2010, Australia.

*Corresponding author. Email: Mick.Ashcroft@austmus.gov.au

Phone: x-61-2-93206475, Fax: x-61-2-93206486

Word count (including text, references, and captions): 7114

\footnotetext{
${ }^{1}$ Current address: New South Wales Rural Fire Service, 16 Carter St, Homebush Bay, NSW, 2127, Australia.

${ }^{2}$ Current address: Pest Management Unit, NSW National Parks and Wildlife Service, 43 Bridge St, Hurstville, NSW, 2220, Australia.

${ }^{3}$ Current address: Department of Biological Sciences, Macquarie University, Sydney, NSW, 2109, Australia.

${ }^{4}$ Current address: Evolution \& Ecology Research Centre, School of Biological, Earth and Environmental Sciences, University of New South Wales, Sydney, NSW, 2052, Australia.
} 
Abstract It is rarely cost-effective to survey invertebrates for use in systematic conservation planning activities. The efficiency of sampling methods needs to be improved, and this is especially important at landscape and regional scales. We investigated two methods that could be used to improve regional scale sampling efficiency using a case study of ants, beetles, flies, bugs, spiders and wasps from the semi-arid Pilbara region of Western Australia. First, Generalised Dissimilarity Models (GDMs) were used to divide the region into landscapes with relatively homogeneous communities and environmental conditions. We found that some of these landscapes were large, and a low sampling density could be employed in these areas due to the low spatial turnover in species. Other landscapes were 1-2 orders of magnitude smaller, and a higher sampling density should be employed to capture the high species turnover and unique species in these areas. Variation of sampling density based on landscape dimensions could vastly improve survey efficiency. Second, we investigated whether one large sample or five small samples were a more efficient method to estimate the species composition of each landscape. We found that five small samples captured a higher proportion of landscape scale species richness for a fixed sampling effort, and was therefore a more efficient method to determine the species composition of the landscape. Combining five small samples also resulted in less sample variability than one large sample, which increases statistical power to detect changes. We concluded that GDM was an effective method to increase sampling efficiency, because it allowed sampling density to vary according to the spatial turnover in species. Using many small samples is a more efficient method to capture the species composition of landscapes than a single large sample with an equivalent sample size.

Keywords Arthropod, Generalized Dissimilarity Model, Monitoring, Semi-arid area, Species richness, Survey design 


\section{Introduction}

Invertebrates are rarely considered in conservation planning due to inadequate knowledge of their distributional patterns and the high cost of surveys. To address this deficiency, it is necessary to increase the cost-effectiveness of surveys and improve the efficiency of sampling methods (Oliver and Beattie, 1996). As most of the expense of invertebrate surveys is associated with sorting and identifying large numbers of specimens (Wilkie et al., 2003), one approach to improve the efficiency of surveys is to minimise the size and number of samples that are required to capture the necessary information.

It is especially important to develop survey methods that are efficient at estimating regional communities, as biases may be introduced when the diversity of larger regions is estimated by combining the samples from a limited number of local sites (Yoccoz et al., 2001). Many studies consider local communities as fundamental ecological units, but there is increasing awareness that these are dynamic and variable in composition due to stochastic extinction, colonisation and dispersal events (Hubbell, 2001; Leibold et al., 2004; Ricklefs, 2008). However, the regional community may be in equilibrium even when the local communities are not (Whittaker et al., 2001). The key question for conservation is not necessarily whether a species can persist at any individual locality, but whether it can persist in the region as a whole (Hanski, 1994). Therefore, it is important to determine how regional communities can best be sampled.

We addressed two aspects of the sampling design for regional invertebrate communities using a case study of arthropods in a semi-arid area. Our first goal was to use Generalised Dissimilarity Models (GDMs; Ferrier et al., 2007) to divide the region into relatively homogeneous landscapes. While many studies define landscapes or regions based on political 
boundaries, such as countries (e.g. Schweiger et al., 2005), or fixed areas, such as $500 \mathrm{~km} \times 500$ km quadrats (e.g. Caley and Schluter, 1997), these may be inefficient as they are not based on ecological attributes. Some areas may be large and homogeneous and can be sampled less intensively than a small area with unique species. We acknowledge that it is desirable to keep the area of each landscape constant when comparing their diversities (Walther et al., 1995; Gotelli and Colwell, 2001; Whittaker et al., 2001), but propose that this is not necessary when inventories are not being compiled for comparative purposes. Instead, GDMs can be used to quantify the turnover in species along environmental gradients, and the models used to define large landscapes in areas with low turnover, and smaller landscapes where there is high turnover. This modelling approach offers the potential to improve the efficiency of future regional scale surveys, because we can vary the intensity of sampling according to the expected turnover in species composition. While landscapes will vary in size, each has a similar amount of species turnover, and can be allocated equal sampling effort, such as the number of invertebrate traps deployed.

The second goal of this study was to examine the trade-off between many small samples and few large samples (as done in other contexts by Rosenzweig et al., 2003; MacKenzie and Royle, 2005; Munoz et al., 2007; Beck and Kitching, 2007), to determine which was more efficient at sampling the composition of each landscape. This trade-off is necessary as the total sampling effort is typically predetermined based on available resources. We used a sub-sampling approach to investigate whether, given a fixed number of invertebrate traps (or samples), it was better to conduct extensive sampling (five sites, one trap per site) or intensive sampling (one site, five traps) to capture the composition of landscape scale invertebrate communities. The better method would sample a higher proportion of total landscape species richness and achieve more 
consistent results when the method was repeated. Extensive sampling (many sites) probably covers a broader range of environmental conditions and the habitat heterogeneity hypothesis suggests this should lead to higher diversity (MacArthur and MacArthur, 1961). However, James (2004) found that the opposite was true for ants in arid rangelands. That is, that multiple traps at a single site captured a higher richness than the same number of traps spread over multiple sites. Our comparison between intensive and extensive sampling investigated this discrepancy.

\section{Materials and Methods}

This study was conducted in, and adjacent to, the Pilbara bioregion, Western Australia (115$121^{\circ} \mathrm{E}, 19-25^{\circ} \mathrm{S}$, Fig. 1). The climate is hot and arid, with mean summer maximum temperatures of approximately $38^{\circ} \mathrm{C}$ and rainfall (mostly summer) of $250-400 \mathrm{~mm}$ per year (Fisher et al., 2004). The elevation gradually increases from sea level on the coast to $500 \mathrm{~m}$ at inland locations, with higher elevations in the Hamersley Range (Fig. 1). There is poor knowledge on the distribution of biodiversity in the Pilbara (Fisher et al., 2004). Invertebrates are known to respond to soil properties and artificial watering points on grazing land (Gollan et al., 2009), but the influence of broad scale climatic factors is unknown.

Arthropods were sampled at 46 sites between $20^{\text {th }}$ May and $3^{\text {rd }}$ June 2006. Each site was sampled for 10 days but start dates varied from $20^{\text {th }}$ May to $24^{\text {th }}$ May for logistical reasons. Five pitfall traps were established in each $20 \mathrm{~m}$ by $20 \mathrm{~m}$ site in a quincunx formation (one trap in each corner and another in the middle). All samples were processed according to standardised sorting protocols (Wilkie et al., 2003). Beetles (Coleoptera), flies (Diptera), ants (Hymenoptera: Formicidae), wasps (Hymenoptera excluding Formicidae and Chalcidoidea), spiders (Araneae) 
and bugs (Hemiptera) were identified to either species (where possible) or morphospecies. All taxonomic assignments are hereafter referred to as 'species', regardless of whether they were assigned morphospecies or formally identified. Voucher specimens were lodged at the Australian Museum.

\subsection{Determining the boundaries of landscapes}

Generalised Dissimilarity Models (GDMs) were developed to classify the Pilbara region into seven distinct landscapes. GDM software for the R statistical software environment can be downloaded from http://www.biomaps.net.au/gdm/, although we used an alternative command line executable provided by Glenn Manion and Simon Ferrier. GDM software calculated the Bray-Curtis dissimilarities $\left(\mathrm{d}_{\mathrm{ij}}\right)$ in species composition between sampled sites (in paired combinations of $\mathrm{i}$ and $\mathrm{j})$, and then derived monotonically increasing functions $\left(f_{\mathrm{k}}\right)$ for each of $\mathrm{p}$ environmental factors using a Generalised Linear Model (GLM) and an exponential link function using the following equation (Ferrier et al., 2007; Overton et al., 2009):

$-\ln \left(1-d_{i j}\right)=b+\sum_{k=1}^{F} \mid f_{k}\left(x_{k t}\right)-f_{k}\left(x_{k \eta 2} \mid\right.$

Two sites with identical environmental conditions have a dissimilarity that is determined by the intercept b (Overton et al., 2009). We then used GDM software to estimate the dissimilarities between other geographic locations in the Pilbara (cells in a raster image) based on their environmental conditions, and used multi-dimensional scaling techniques to classify the study area into landscapes that were relatively homogeneous in environmental conditions and species 
composition (Ferrier et al., 2007). The number of landscapes (seven) was selected as a tradeoff between more landscapes (less samples within each landscape) and less landscapes (more environmental variability within each landscape).

Environmental predictors used to generate GDM models were BIOCLIM predictors 1-7, 10-13, 15-16, 20-23, 28-29 and 31-32 (Houlder et al., 2003). Some predictors were not considered because they had low spatial variability (e.g. no rainfall during driest periods) or because the classification into wettest or driest quarters led to spurious results. The selected predictors included a range of climatic variables including mean annual temperature, annual precipitation, annual mean radiation, annual mean moisture index and various estimates of extreme conditions and climatic variability (Table 1). Raster grids of each BIOCLIM predictor were developed using ANUCLIM v5.1 (Houlder et al., 2003) and a 250 m Digital Elevation Model (DEM) for Australia available from Geoscience Australia. One GDM was produced for each of the six target taxa, and another using the combined dataset. GDM software automatically removed environmental factors that did not significantly affect the turnover in species composition. The influence of each factor was assessed based on the range of the partial response graph (Overton et al., 2009).

\subsection{Comparison between intensive and extensive sampling}

Five groups of five sites were chosen to compare intensive and extensive sampling using a subsampling approach (e.g. Franco et al., 2007). The sites were selected so that they predominately belonged to the same landscapes as defined by the GDM models, and are hereafter assumed to be in the same landscape. Some discrepancies were unavoidable due to different landscape 
boundaries for different taxa (see results for further details). In addition, only 43 of the 46 sites were considered, as some traps were damaged at three sites.

Intensive sampling was designed to represent one large sample from a single site, and was implemented by pooling data from all five pitfall traps at one site. Extensive sampling was designed to represent many small samples, and was implemented by pooling data from one pitfall trap at each of the five sites in a given landscape. There were five replicates for each sampling method in each landscape. For intensive sampling, each replicate consisted of five traps from a different site, while for extensive sampling each replicate was a different trap from each of the five sites (Fig. 2).

We investigated whether intensive (five traps from one site) or extensive (one trap from each of five sites) sampling gave a better representation of the total landscape community (all 25 traps - five traps at each of five sites). This was achieved by determining the average proportion of landscape abundance and richness obtained by the five replicates of each of the sampling methods. The average abundance for both intensive and extensive samples would obviously be $20 \%$ of the total landscape abundance (each replicate has five of the 25 traps), and so this result was ignored. The average richness could vary between $20 \%$ (all replicates had unique species) to $100 \%$ (all replicates had all species). Higher values indicated that the method was more efficient at sampling the landscape scale community, as a greater proportion of the landscape scale richness was obtained using $20 \%$ of the trapping effort. The difference in results between intensive and extensive sampling methods was tested for significance using a two-sided paired Student's t-test.

The standard deviation of the abundance and richness of intensive and extensive samples was also determined and tested for significant differences as above. A low standard deviation 
indicated that each of the five replicates produced similar results, and were a more consistent representation of the landscape community. A high standard deviation indicated that each replicate produced different results, and this higher sampling variability would decrease the statistical power of monitoring programmes to detect change (e.g. Downes et al., 2002).

\subsection{Validation of methodology}

The tests between intensive and extensive sampling relied on two key assumptions that were subsequently verified. First, the tests were based on the assumption that all five sites in each landscape were similar in species composition, and hence are part of the same landscape scale community. Landscapes were chosen based on the GDM models, but these were verified by producing a non-metric multidimensional scaling (MDS) plot (Primer v5.2.9, Bray-Curtis dissimilarity, presence/absence data; Clarke and Warwick, 1994). The MDS plot was used to visually verify the five sites (five intensive samples) from the same landscape clustered together, and to compare their composition with the five extensive samples from the same landscape. We also used ANOSIM (Primer v5.2.9; Clarke and Warwick, 1994) to verify there were statistically significant differences between the communities in each landscape. We only included the 25 intensive samples in ANOSIM - that is, the pooled samples from the five traps at each of the 25 sites. Only the combined dataset was used to produce an MDS plot and conduct ANOSIM, not the six individual taxa.

Second, any invertebrate study is complicated by sampling adequacy (Coddington et al., 2009). For example, there are typically many singletons in any sample, and further sampling effort would obtain a higher species richness (Fisher et al., 1943). Therefore, we used linear 
regression to establish the relationship between the proportion of landscape richness obtained by intensive and extensive sampling and sample abundance. These relationships were used to estimate the effect that larger sample sizes would have on our results.

\section{Results}

\subsection{The spatial distribution of landscape scale communities}

The environmental factors that were most frequently selected in the seven GDM models (six models for individual taxa plus one for all taxa) were mean diurnal temperature range (BIOCLIM parameter 2), lowest period radiation (parameter 22), isothermality (parameter 3), and annual mean radiation (parameter 20). Each of these four predictors was selected in at least five of the seven models, and their large influence on the results was further confirmed by the high ranges on the partial responses graphs (Overton et al., 2009; Table 1). Mean annual temperature (parameter 1), minimum temperature of the coolest period (parameter 6), precipitation seasonality (parameter 15) and maximum temperature of the warmest period (parameter 5) had a large influence in one or two models, but were excluded altogether from four or five of the seven models. Predictors associated with precipitation and moisture were selected less often than those associated with temperature and radiation (32\% versus $48-50 \%$ ), and typically had lower coefficients (Table 1).

The landscape maps produced by GDM each showed a distinct change in composition from coastal to inland areas (Fig. 3). A northwest to southeast feature was prominent in all images apart from bugs, corresponding with the location of the Hamersley Range. There were distinct 
landscapes corresponding with locations of higher elevation, and many communities only existed on one side of the mountain range or the other. The smallest landscapes in the seven models ranged from $225-10,272 \mathrm{~km}^{2}$, while the largest ranged from $45,440-111,102 \mathrm{~km}^{2}$. Some landscapes were comprised of multiple fragments with the same community on both sides of the Hamersley range.

\subsection{Comparing intensive and extensive sampling}

Five landscapes, each containing five sites, were selected to compare intensive and extensive sampling. In each landscape, each site was categorised identically to the other four in at least five of the seven GDM models. Some variations were unavoidable due to differences in landscape boundaries between taxa (Fig. 3). Two of the selected landscapes were found in coastal areas (Fig. 3, large squares, small triangles), two in inland areas (large triangles, circles), and one at higher elevation (small squares). One of the inland landscapes was spatially discontinuous (large triangles), reflecting the output from models for ants, beetles and flies (Fig. 3). The differences between intensive and extensive sampling were similar in all landscapes, and were apparently unaffected by landscape size, continuity, or location.

The entire survey obtained 17,476 specimens and 592 species. The abundance captured in the five selected landscapes varied from 1,512 in the high elevation landscape, to 3,286-3,986 in the coastal landscapes. The inland landscapes had abundances of 2,102-2,260. The total richness of the landscapes showed a similar trend, and varied from 125 species in the high elevation landscape to 159 in each of the coastal landscapes. The inland landscapes had 146-147 species. There was an average of 1,891 ants per landscape, 339 flies, 172 bugs, 103 beetles, 80 wasps, 
and 44 spiders. Each landscape contained an average of 38 ant species, and 19-24 species of each of the other taxa.

Overall ( $\mathrm{n}=6$ taxa $\times 5$ landscapes $=30)$, extensive sampling captured a significantly greater portion of landscape scale species richness than intensive sampling (paired t-test, $\mathrm{P}<0.001$ ), and was less variable in terms of both abundance $(\mathrm{P}<0.001)$ and richness $(\mathrm{P}=0.002)$. There was less statistical power to detect differences using individual taxa ( $\mathrm{n}=5$ landscapes), but extensive sampling still captured significantly $(\mathrm{P}<0.05)$ higher richness for spiders, flies, beetles and ants, and was significantly less variable in abundance (wasps, bugs, flies) and richness (flies, ants). Of the nine insignificant results with individual taxa, four had $\mathrm{P}$ values less than 0.1 . On average across the five landscapes, extensive sampling captured higher richness than intensive sampling for all taxa, was less variable in terms of abundance, and less variable in richness for all taxa apart from bugs (Fig. 4).

The difference in richness between intensive and extensive sampling was affected by the abundance of invertebrates sampled in the landscape. As the sample abundance increased, the proportion of landscape richness obtained by intensively sampling one site also increased. However, the proportion of richness obtained by extensive sampling increased at a greater rate, and therefore the difference between intensive and extensive sampling became greater (Fig. 5a). While taxa varied in the proportion of richness obtained by intensive and extensive sampling (Fig. 4), these differences could be explained by variations in abundance (Fig. 5b, c).

ANOSIM results confirmed that the samples from each landscape generally had significantly different species compositions from other landscapes (Table 2), although one of the inland landscapes (circles in Fig. 3) was not significantly different from three of the four landscapes ( $\mathrm{P}>0.05$ ). Overall, the results were significant (global $\mathrm{R}=0.33, \mathrm{P}<0.01$ ), verifying 
the validity of the landscapes we selected using GDM. The MDS plot also confirmed visually that the samples from each landscape clustered together, although this effect was stronger for the extensive samples than the intensive samples (Fig. 6).

\section{Discussion}

\subsection{How should sampling be distributed across a region to maximize sampling efficiency?}

Geographically uniform sampling of the Pilbara bioregion would be an inefficient method to capture the regional invertebrate community. Our Generalised Dissimilarity Models (GDMs) suggested that there were relatively small landscapes in coastal and high elevational locations, and these need a higher sampling density to capture the high species turnover in these areas. These small landscapes with unique conditions may also contain many rare and restricted species. Conversely, there were larger landscapes on the inland plains, and a lower sampling intensity could be used in these areas to decrease redundancy and increase sampling efficiency. The landscape sizes varied by 1-2 orders of magnitude for each GDM we produced, demonstrating the potential variations in sampling density that could be employed.

Site base methods of designing surveys, such as Survey Gap Analysis (SGA), can also be performed with GDM using the concept of Environmental Diversity, and would result in similar variations in sampling intensity (Faith and Walker, 1996; Ferrier et al., 2007). However, as discussed in the introduction, individual sites are often dynamic and we were more concerned with sampling the regional or landscape scale communities than the species assemblage at any individual site. The intent of our methods was to pool samples to define the landscape or regional 
scale communities, whereas SGA is generally used to determine sites that will be analysed individually.

We found that landscape delineations were complicated by variations between the different taxa. For example, bugs were more homogeneous in inland areas (a few large landscapes indicating low turnover) than coastal areas (many small landscapes indicating higher turnover). In contrast, wasps, ants and beetles were relatively homogenous in coastal areas, and had higher turnover inland and at higher elevation. This highlights the dangers of using one taxon to determine landscapes for all, as observed previously for site based methods (Faith and Walker, 1996). Similarly, it could not be expected that politically defined regions or catchments could sufficiently delineate landscapes for all taxa, especially when there are similar environmental conditions on both sides of an arbitrarily defined boundary.

In reality, the boundaries between landscapes are not hard and fixed, but will vary temporally and from taxon to taxon. The delineation of landscapes should be viewed as a tool to approximate 'independent' areas and improve sampling efficiency, but there will no doubt be species that span part of one landscape and part of another. The landscape delineations we created could be used so that the species composition within each landscape could be independently monitored over time, or the results from each landscape could be combined to estimate the regional community. Monitoring individual sites may still be necessary for populations of range restricted species with specific habitat requirements, but landscape scale sampling will be beneficial where neutral drift or metapopulation dynamics result in dynamic local communities and stochastic extinctions and colonisations at individual sites (Hubbell, 2001; Leibold et al., 2004; Ricklefs, 2008). Even if the landscape boundaries we defined are not perfectly distinct, they still provide approximate borders for areas with relatively similar species 
composition, and conducting analysis using these landscapes will reduce the effect of local site dynamics.

While we determined the location of landscapes using only environmental factors, it is interesting to note that there were many unique landscapes on one side of the Hamersley range or the other. While it is possible that this reflects a temperature gradient or rain shadow, it is also possible that the mountain range is acting as a barrier to dispersal. In contrast, we also determined that some landscapes had disjointed distributions, with distinct fragments from the same landscape observed on either side of the Hamersley Range. This supports the notion that similar communities occur in areas with similar environmental conditions, and suggests that the Hamersley range may not be a barrier for all species.

\subsection{The trade-off between many small samples and few large samples}

The trade-off between many small samples and few large samples is frequently discussed and has different implications in a number of contexts (e.g. Rosenzweig et al. 2003; MacKenzie and Royle 2005; Munoz et al. 2007; Beck and Kitching 2007). Our study addressed a specific example of this trade-off-whether it was better to place five traps at one site, or five traps at different sites, in order to sample landscape scale communities. In the context of our study we were trying to maximize how many species would be captured with a given sampling effort, as opposed to trying to estimate the asymptotic richness of any site, landscape or region (Colwell and Coddington, 1994).

We found that extensive sampling (five sites, one trap per site) was consistently more efficient than intensive sampling (one site, five traps) in the context of landscape scale sampling. 
First, extensive sampling captured more component species of the landscape scale community than the same effort using intensive sampling. This is probably due to higher environmental diversity and less spatial autocorrelation when the traps are placed at different sites (see next section). Second, the replicates for extensive sampling were less variable in terms of both abundance and richness than intensive sampling, and this is probably also because the extensive samples were less spatially autocorrelated and more indicative of the overall landscape. The higher richness captured by extensive sampling increases sampling efficiency, while the reduced sampling variance increases the statistical power of monitoring programmes to detect change. In effect, if different samples taken from the same landscape at the same time are highly variable, then this will disguise any temporal trends that occur over multiple sampling periods. Finally, the five extensive replicates were more similar in species composition than the intensive replicates, and this will also make it easier to detect temporal trends in composition. This reduced variability in species composition is consistent with the higher richness obtained by extensive sampling, which indicates that the extensive samples are a more complete sample of the landscape scale community. All results suggest that placing five traps at one site produced a result that was less indicative of the landscape scale community than spreading the same sampling effort across five sites.

It is unlikely that anyone would conduct a survey by placing five traps at one site, or one trap at five sites, as the sample size would be too small to detect trends. The actual trade-off may be between, in our case for example, 46 sites with five traps at each, and 230 sites with one trap at each. The main advantage of surveying 46 sites with five traps per site is that it provides a larger, and replicated, sample of local communities. However, Ricklefs $(2004,2008)$ argues that local communities only exist in the case of distinct areas of unique environmental conditions, 
and therefore the benefits of sampling local communities are not always applicable. Surveying 230 sites with one trap per site provides more data to delineate landscapes, increases the number of species we expect to obtain, and provides less variable estimates of landscape and regional abundance, richness and composition. It is common practice to thoroughly sample each locality in a survey, but we recommend changing to a more extensive sampling method so that we increase our knowledge on the spatial and temporal aspects of landscape and regional communities. Intensive local scale sampling should be employed for 'local species' that are dispersal limited (Poiani et al., 2000), but would perform poorly for species that use the whole landscape but only occupy a limited portion at any one time (MacKenzie and Royle, 2005).

We observed a high proportion of species in low abundance, and this suggests that 10 days sampling was insufficient to capture all species at each site, or in each landscape. However, this does not invalidate the comparison between intensive and extensive sampling. Our results showed that larger samples increased the benefits of extensive sampling over intensive sampling. Therefore, we expect increased sampling effort to increase the advantage of extensive sampling. It should also be noted that obtaining a complete species inventory of invertebrates on multiple occasions is beyond the resources available to most monitoring programmes. Capturing the dominant species in the community on multiple occasions is feasible, and this level of monitoring would be more beneficial for many management purposes than a complete inventory taken at only one point in time.

Our comparison between extensive and intensive sampling suggests that it is better to have many small, spatially separated samples, than a smaller number of large or clustered samples. This reflects a spatial component to sampling efficiency, but there may also be a temporal 
component. The efficiency of sampling programmes may also be increased by sampling at different times of the year, or in different years (e.g. Coddington et al., 2006).

\subsection{Factors contributing to the results}

A number of alternative theories and factors can explain why extensive sampling was more efficient than intensive sampling, although they are not mutually exclusive. First, if sites within the landscapes varied in environmental conditions, then niche theory suggests this would cause variations in composition and abundance. Extensive sampling would then sample a greater range of environmental conditions than intensive sampling, and hence would be expected to encounter different communities and obtain higher diversity (MacArthur and MacArthur, 1961). Niche factors will have affected some species or taxonomic groups in our study, but we minimised the impact by using GDM to select landscapes that were relatively homogeneous in environmental conditions. Results from ANOSIM and our MDS plot showed the composition of each site in each landscape was relatively constant (clustered), and hence compositional and environmental differences between sites were kept to a minimum.

It is also possible that nearby sites were similar in species composition due to random drift in neutral models (Hubbell, 2001) or with other factors that lead to spatial autocorrelation (e.g. dispersal). Five traps at one site would be a spatially autocorrelated sample, and would not be expected to sample all species from the landscape. It is difficult to distinguish spatial autocorrelation from environmental factors, as both make similar predictions when nearby sites are more similar in environmental conditions than distant sites. It should be noted, however, that some landscapes were fragmented, with different patches separated by the Hamersley range. In 
this situation, similarity in species composition cannot be adequately explained simply by clustering due to dispersal.

All the above factors suggest that higher diversity should be obtained by extensive sampling (as we found) but it is interesting to note that James (2004) found the opposite result. That is, that a higher diversity could be obtained by intensively sampling one site. It is important to note that methodological differences between his study and ours may have contributed to this difference. We had five pitfall traps at all sites and analysed six different taxa by pooling different combinations of traps. James (2004) varied the number of traps present at each site and only analysed ants. If the number of traps present affects biotic processes such as competition (as suggested by James), our results might not be valid when trapping pressure varies. Alternatively, James' (2004) results may not be indicative if the higher sampling density sites were coincidentally higher in biodiversity due to, for example, unmeasured environmental factors. In addition, only ants were analysed by James (2004), and traps were deliberately placed to avoid foraging trails. This may have introduced a sampling bias if it was more difficult to avoid these runs when there were more traps at a site.

Another factor that may have influenced our results is the non-replacement nature of trapping. Pitfall traps remove individuals from the local community, and this may allow the abundance to fall below the carrying capacity and/or release competitive exclusion of species (James, 2004; Gibb, 2005). Both these factors increase the probability that transient, competitively inferior, or colonising species can occupy the site. This effect would increase as intensive sampling was conducted over longer periods of time, and may mean that individuals captured are not part of the community that normally occupies the site. Nevertheless, we do not 
believe this affected our results as trap catches were low in the selected landscapes (average of 10.5 individuals per trap per day).

\section{Conclusions}

There is a need to collect empirical data on the spatial and temporal variability of invertebrate communities at the landscape and regional scales. Proposed data collection methodologies should be compatible with the scale of analysis, and with the scale of ecological processes that are responsible for the distributional patterns in biodiversity (Ricklefs, 1987). We propose that many, widespread, small samples are needed to study landscape scale communities, even if this reduces the replication or completeness of samples at the local scale. Local scale samples provide a biased estimate of landscape scale communities, as they are spatially autocorrelated and do not cover the full range of environmental conditions.

Using GDM to define the boundaries of landscapes ensures that they are based on ecologically meaningful attributes rather than arbitrary political boundaries or regions of fixed size and shape. Allowing variations in the size of landscapes (Fig. 3) allows the sampling strategy to capture all communities present in the region, and varying sampling density according to landscape size can improve sampling efficiency. Using GDM allows samples to be pooled over landscapes that have relatively homogeneous communities, and this reduces variability and gives monitoring programmes more chance of detecting change in those landscapes.

The higher species richness obtained by extensive sampling indicates that the sampling scheme is more optimal—capturing a greater proportion of landscape scale biodiversity for the equivalent sampling effort. The lower variance in abundance and richness indicates there is less 
natural variability amongst replicates-increasing the statistical power to detect meaningful changes in biodiversity. Therefore, the results of our study reinforce Gillison and Liswanti's (2004) suggestion that it is more efficient for landscape scale monitoring to have many small samples than a few large samples.

\section{Acknowledgements}

This project was undertaken as part of the Australian Museum - Rio Tinto partnership and BioMaps and was funded by the Rio Tinto WA Future Fund. Thanks are given to Georgina Brown, Louise Kampen and Alex Hegedus for their assistance in the field and with sorting. We wish to acknowledge Derek Smith (Formicidae), Helen Smith (Araneae), Georgina Brown (Coleoptera and Hemiptera) and Michael Elliott (Hymenoptera) for taxonomic identification. We also thank Chris Reid, Dan Bickel, David McAlpine, Shane McEvey for their taxonomic advice, and Lance Wilkie for assistance with survey design. GDM software was courteously supplied by Simon Ferrier and Glenn Manion.

\section{References}

Beck, J., Kitching, I.J., 2007. Estimating regional species richness of tropical insects from museum data: a comparison of a geography-based and sample-based methods. Journal of Applied Ecology 44, 672-681.

Caley, M.J., Schluter, D., 1997. The relationship between local and regional diversity. Ecology 78, 70-80. 
Clarke, K.R., Warwick, R.M., 1994. Change in Marine Communities: An Approach to Statistical Analysis and Interpretation. Bourne Press Limited, Bournemouth.

Coddington, J.A., Agnarsson, I., Miller, J.A., Kuntner, M., Hormiga, G., 2009. Undersampling bias: the null hypothesis for singleton species in tropical arthropod surveys. Journal of Animal Ecology 78, 573-584.

Colwell, R.K., Coddington, J.A., 1994. Estimating terrestrial biodiversity through extrapolation. Philosophical Transactions of the Royal Society of London B 345, 101-118.

Downes, B.J., Barmuta, L.A., Fairweather, P.G., Faith, D.P., Keough, M.J., Lake, P.S., Mapstone, B.D., Quinn, G.P., 2002. Monitoring Ecological Impacts: Concepts and Practice in Flowing Waters. Cambridge University Press, New York.

Faith, D.P., Walker, P.A., 1996. Environmental diversity: on the best-possible use of surrogate data for assessing the relative biodiversity of sets of areas. Biodiversity and Conservation 5, 399-415.

Ferrier, S., Manion, G., Elith, J., Richardson, K., 2007. Using generalized dissimilarity modelling to analyse and predict patterns of beta diversity in regional biodiversity assessment. Diversity and Distributions 13, 252-264.

Fisher, A., Hunt, L., James, C., Landsberg, J., Phelps, D., Smyth, A., Watson, I., 2004. Review of total grazing pressure management issues and priorities for biodiversity conservation in rangelands: A resource to aid NRM planning. Desert Knowledge CRC and Tropical Savannas Management CRC, Alice Springs.

Fisher, R.A., Corbet, A.S., Williams, C.B., 1943. The relation between the number of species and the number of individuals in a random sample of an animal population. Journal of Animal Ecology 12, 42-58. 
Franco, A.M.A., Palmeirim, J.M., Sutherland, W.J., 2007. A method for comparing effectiveness of research techniques in conservation and applied ecology. Biological Conservation 134, 96-105.

Gibb, H., 2005. The effect of a dominant ant, Iridomyrmex purpureus, on resource use by ant assemblages depends on microhabitat and resource type. Austral Ecology 30, 856-867.

Gillison, A.N., Liswanti, N., 2004. Assessing biodiversity at landscape level in northern Thailand and Sumatra (Indonesia): the importance of environmental context. Agriculture, Ecosystems and Environment 104, 75-86.

Gollan, J.R., Ashcroft, M.B., Cassis, G., Donnelly, A.P., Lassau, S.A., 2009. Testing common habitat-based surrogates of invertebrate diversity in a semi-arid rangeland. Biodiversity and Conservation 18, 1147-1159.

Gotelli, N.J., Colwell, R.K., 2001. Quantifying biodiversity: procedures and pitfalls in the measurement and comparison of species richness. Ecology Letters 4, 379-391.

Hanski, I., 1994. A practical model of metapopulation dynamics. Journal of Animal Ecology 63, $151-162$.

Houlder, D., Hutchinson, M., Nix, H., McMahon, J., 2003. ANUCLIM 5.1 User’s Guide. Centre for Resource and Environmental Studies, Australian National University, Canberra.

Hubbell, S.P., 2001. The Unified Neutral Theory of Biodiversity and Biogeography. Princeton University Press, Princeton.

James, C.D., 2004. Trapping intensities for sampling ants in Australian rangelands. Austral Ecology 29, 78-86.

Leibold, M.A., Holyoak, M., Mouquet, N., Amarasekare, P., Chase, J.M., Hoopes, M.F., Holt, R.D., Shurin, J.B., Law, R., Tilman, D., Loreau, M., Gonzalez, A., 2004. The 
metacommunity concept: a framework for multi-scale community ecology. Ecology Letters 7, 601-613.

MacArthur, R.H., MacArthur, H.J., 1961. On bird species diversity. Ecology 42, 594-598.

MacKenzie, D.I., Royle, J.A., 2005. Designing occupancy studies: General advice and allocating survey effort. Journal of Applied Ecology 42, 1105-1114.

Munoz, F., Couteron, P., Ramesh, B.R., Etienne, R.S., 2007. Estimating parameters of neutral communities: from one single large to several small samples. Ecology 88, 2482-2488.

Oliver, I., Beattie, A.J., 1996. Designing a cost-effective invertebrate survey: a test of methods for rapid assessment of biodiversity. Ecological Applications 6, 594-607.

Overton, J.McC., Barker, G.M., Price, R., 2009. Estimating and conserving patterns of invertebrate diversity: a test case of New Zealand land snails. Diversity and Distributions $15,731-741$.

Poiani, K.A., Richter, B.D., Anderson, M.G., Richter, H.E., 2000. Biodiversity conservation at multiple scales: Functional sites, landscapes and networks. BioScience 50, 133-146.

Ricklefs, R.E., 1987. Community diversity: Relative roles of local and regional processes. Science 235, 167-171.

Ricklefs, R.E. 2004. A comprehensive framework for global patterns in biodiversity. Ecology Letters 7, 1-15.

Ricklefs, R.E., 2008. Disintegration of the ecological community. American Naturalist 172, 741750.

Rosenzweig, M.L., Turner, W.R., Cox, J.G., Ricketts, T.H., 2003. Estimating diversity in unsampled habitats of a biogeographical province. Conservation Biology 17, 864-874. 
Schweiger, O., Maelfait, J-P., Van Wingerden, W., Hendrickx, F., Billeter, R., Speelmans, M., Augenstein, I., Aukema, B., Aviron, S., Bailey, D., Bukacek, R., Burel, F., Diekötter, T., Dirksen, J., Frenzel, M., Herzog, F., Liira, J., Roubalova, M., Bugter, R., 2005. Quantifying the impact of environmental factors on arthropod communities in agricultural landscapes across organizational levels and spatial scales. Journal of Applied Ecology 42, 1129-1139.

Walther, B.A., Cotgreave, P., Price, R.D., Gregory, R.D., Clayton, D.H., 1995. Sampling effort and parasite species richness. Parasitology Today 11, 306-310.

Whittaker, R.J., Willis, K.J., Field, R., 2001. Scale and species richness: towards a general, hierarchical theory of species diversity. Journal of Biogeography 28, 453-470.

Wilkie, L., Cassis, G., Gray, M., 2003. A quality control protocol for terrestrial invertebrate biodiversity assessment. Biodiversity and Conservation 12, 121-146.

Yoccoz NG, Nichols JD, Boulinier T (2001) Monitoring of biological diversity in space and time. Trends Ecol Evol 16:446-453. 


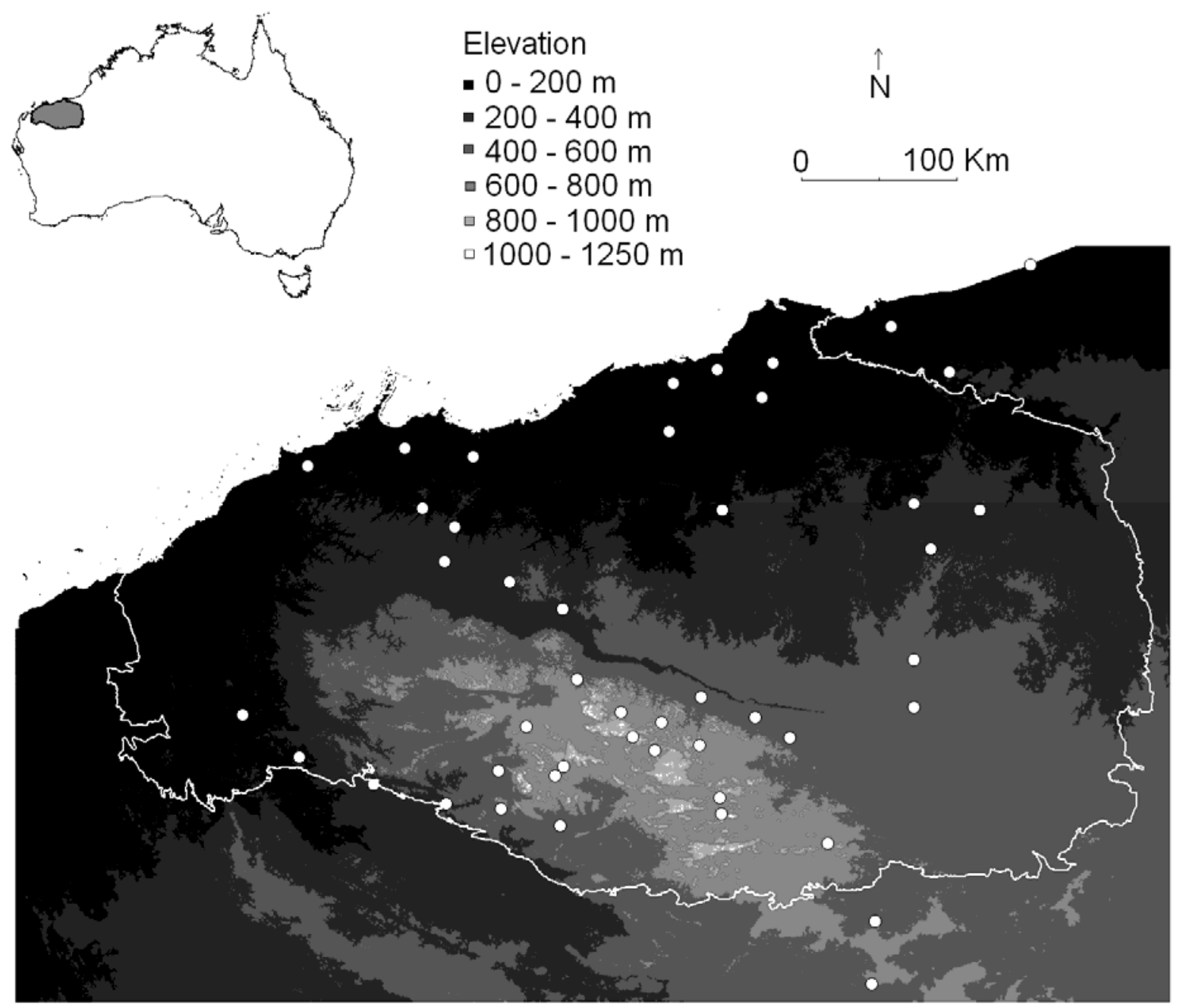

Fig. 1. The grayscale image illustrates the elevation of the study area in $200 \mathrm{~m}$ categories. White dots indicate the sites within, or marginally outside, the Pilbara bioregion where arthropods were collected. The northwest to southeast region of highest elevation is the Hamersley Range. 

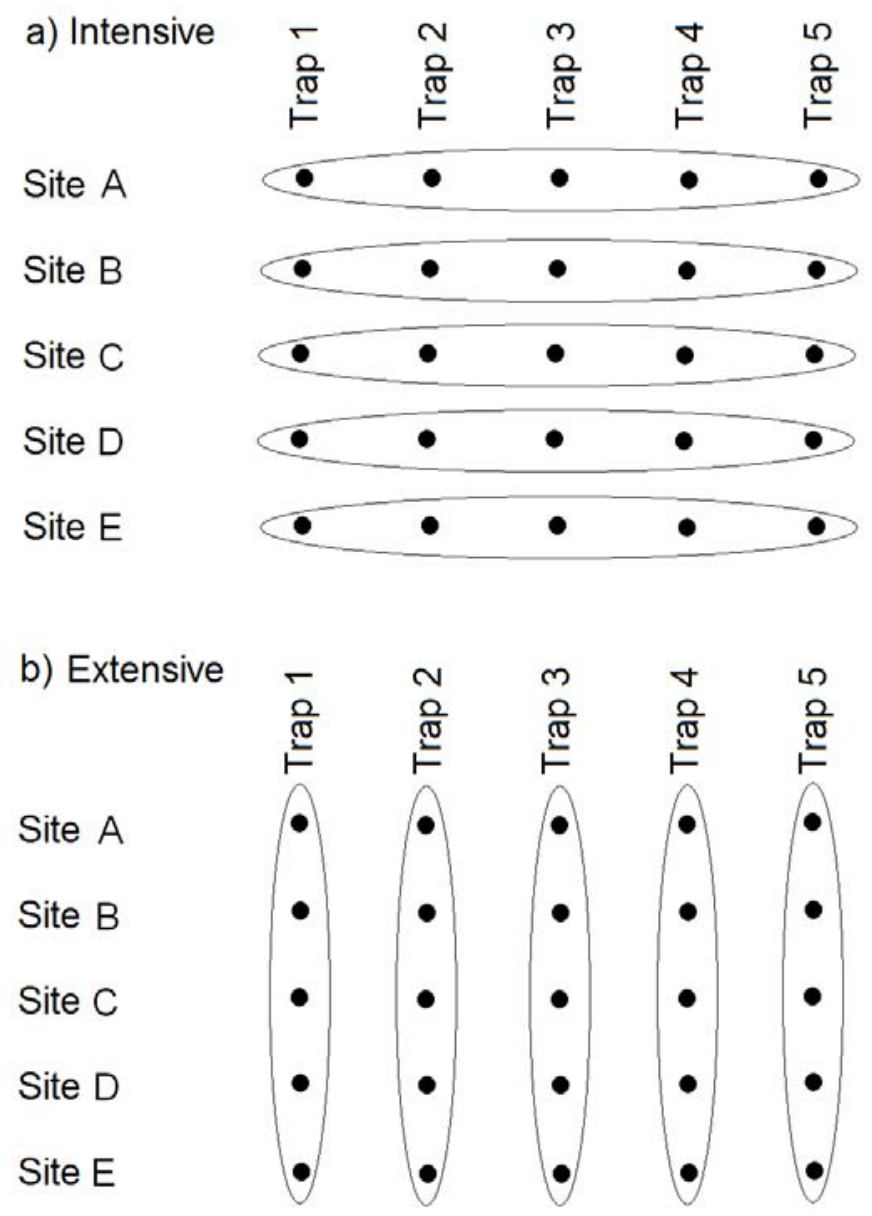

Fig. 2. Each landscape we analysed contained 25 traps-five sites with five traps each. The ovals surround the pitfall traps that were pooled for the intensive (a) and extensive (b) sampling methods. The intensive method involves sampling five traps from one site, while the extensive method involves sampling one trap from each of five sites. In each case there are five replicates—each represented by a separate oval. 

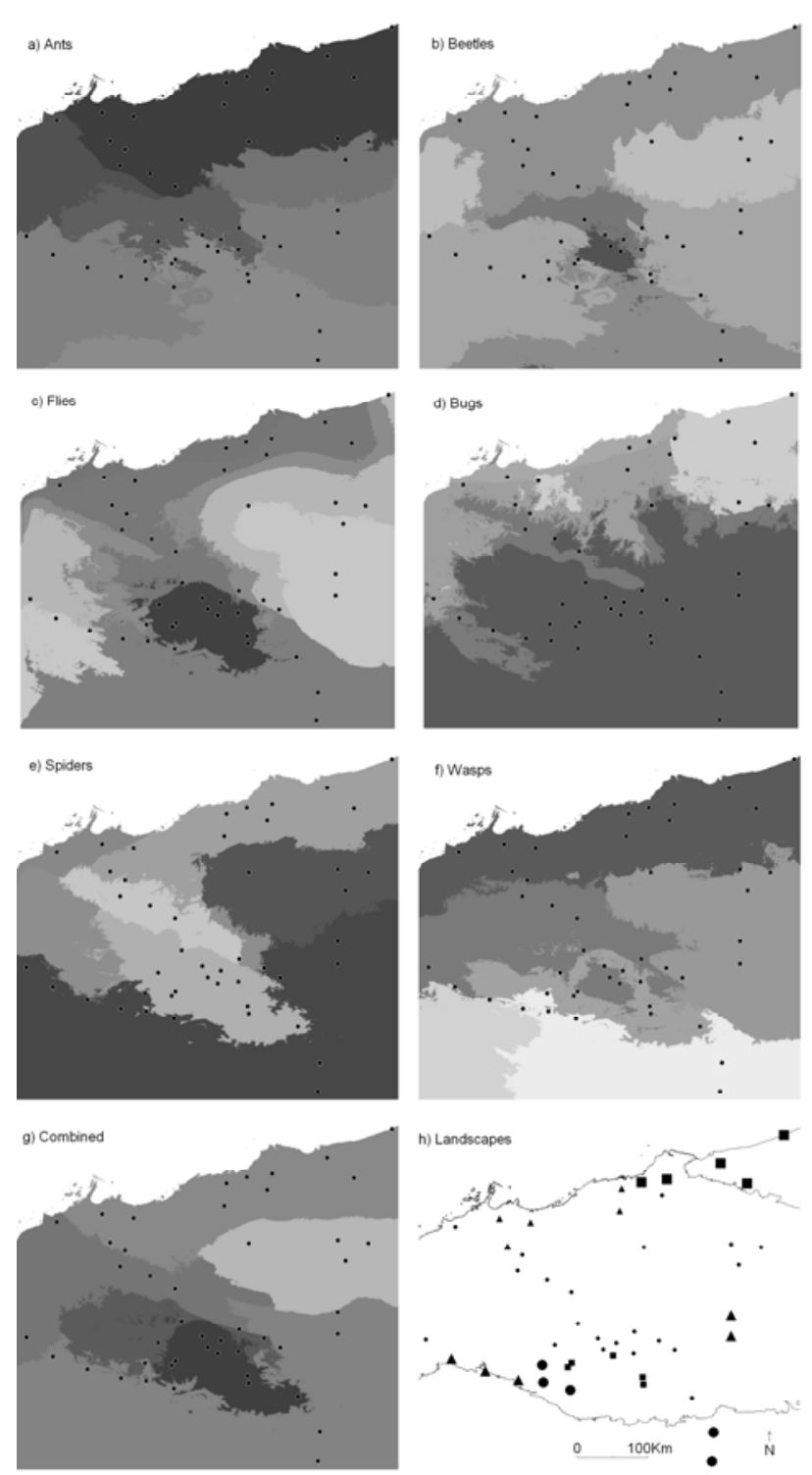

Fig. 3 (grey scale for printed edition). Seven landscapes were defined for each taxonomic group using Generalised Dissimilarity Modelling (GDM). Each landscape is relatively homogenous in terms of species composition and environmental conditions. The more similar in colour two different landscapes are (using RGB composite), the more similar in species composition they are. This figure shows the output in greyscale to indicate the boundaries of landscapes only. The RGB colour image is available in the on-line version. The squares, triangles and large circles in panel h each illustrate a set of five sites that were grouped to form a landscape for comparing sampling methods. Small circles were not selected as part of any landscape. 

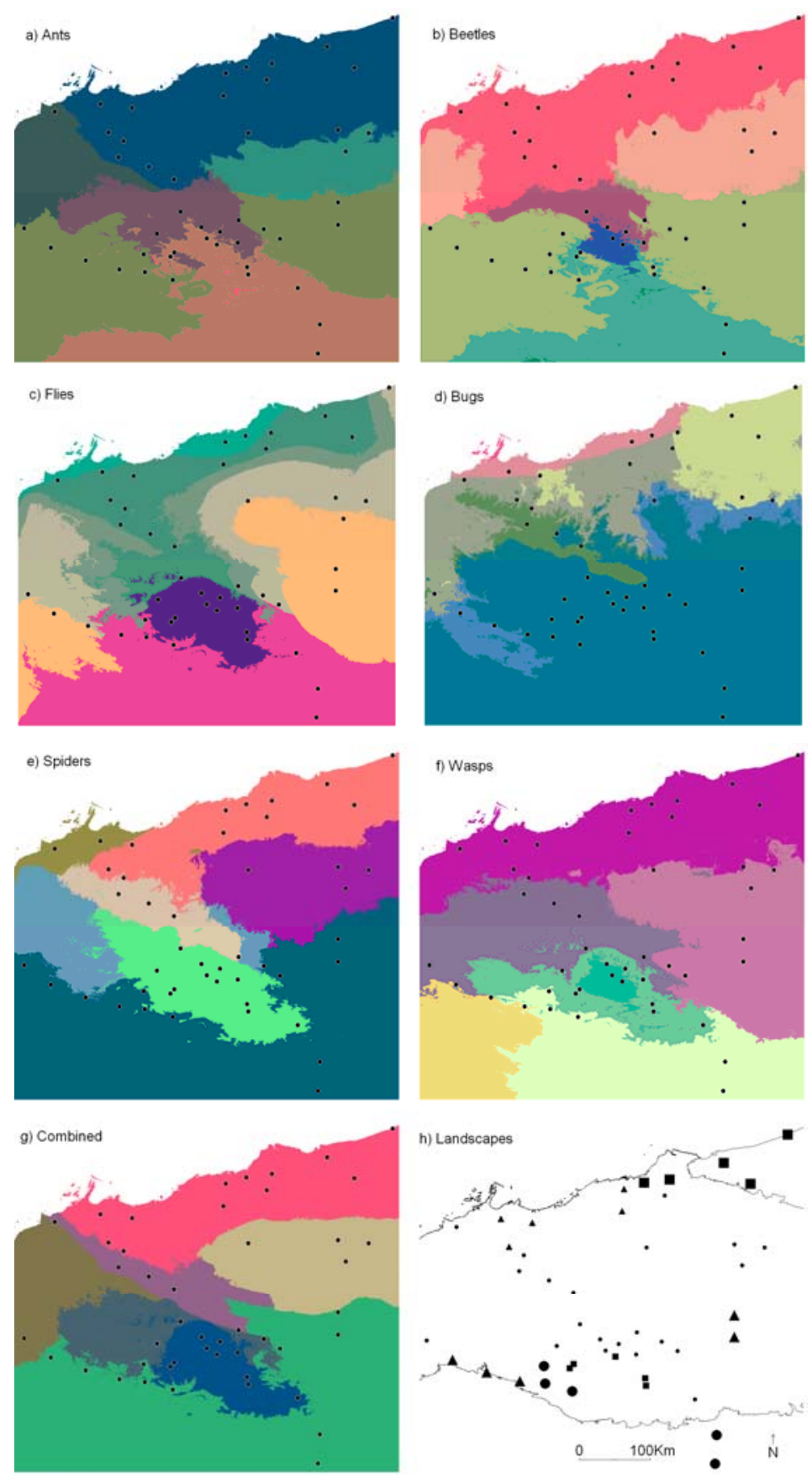

Fig. 3 (colour for online edition). Seven landscapes were defined for each taxonomic group using Generalised Dissimilarity Modelling (GDM). Each landscape is relatively homogenous in terms of species composition and environmental conditions. The more similar in colour two different landscapes are (using RGB composite), the more similar in species composition they are. The squares, triangles and large circles in panel h each illustrate a set of five sites that were grouped to form a landscape for comparing sampling methods. Small circles were not selected as part of any landscape. 

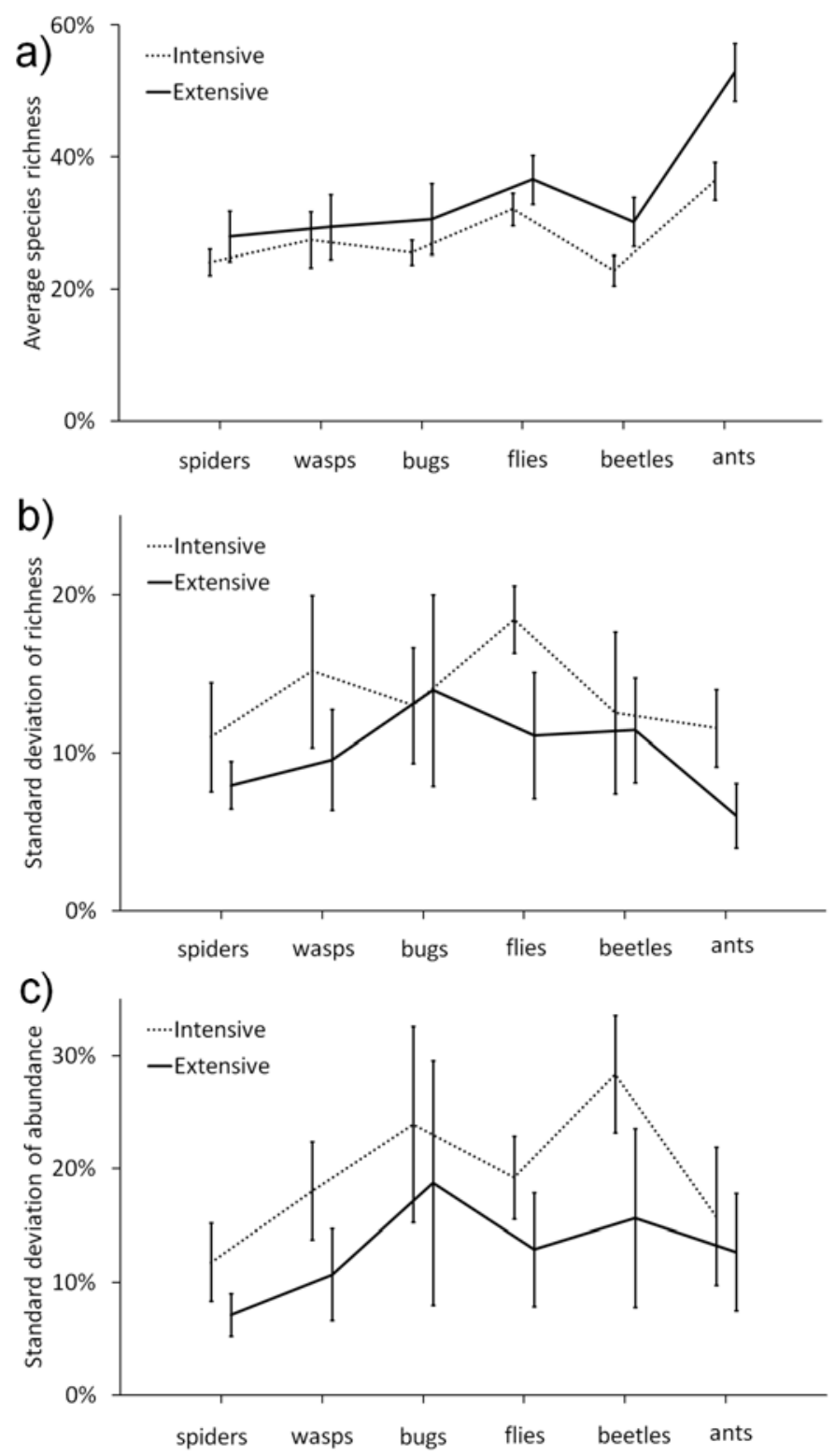

Fig. 4. Panel (a) illustrates the average percentage of landscape scale richness obtained using intensive (five traps at one site) and extensive (one trap at each of five sites) sampling. Panels (b) and (c) show the average standard deviation in richness and abundance respectively, where a low standard deviation is desired as it indicates replicate samples give more consistent results. 

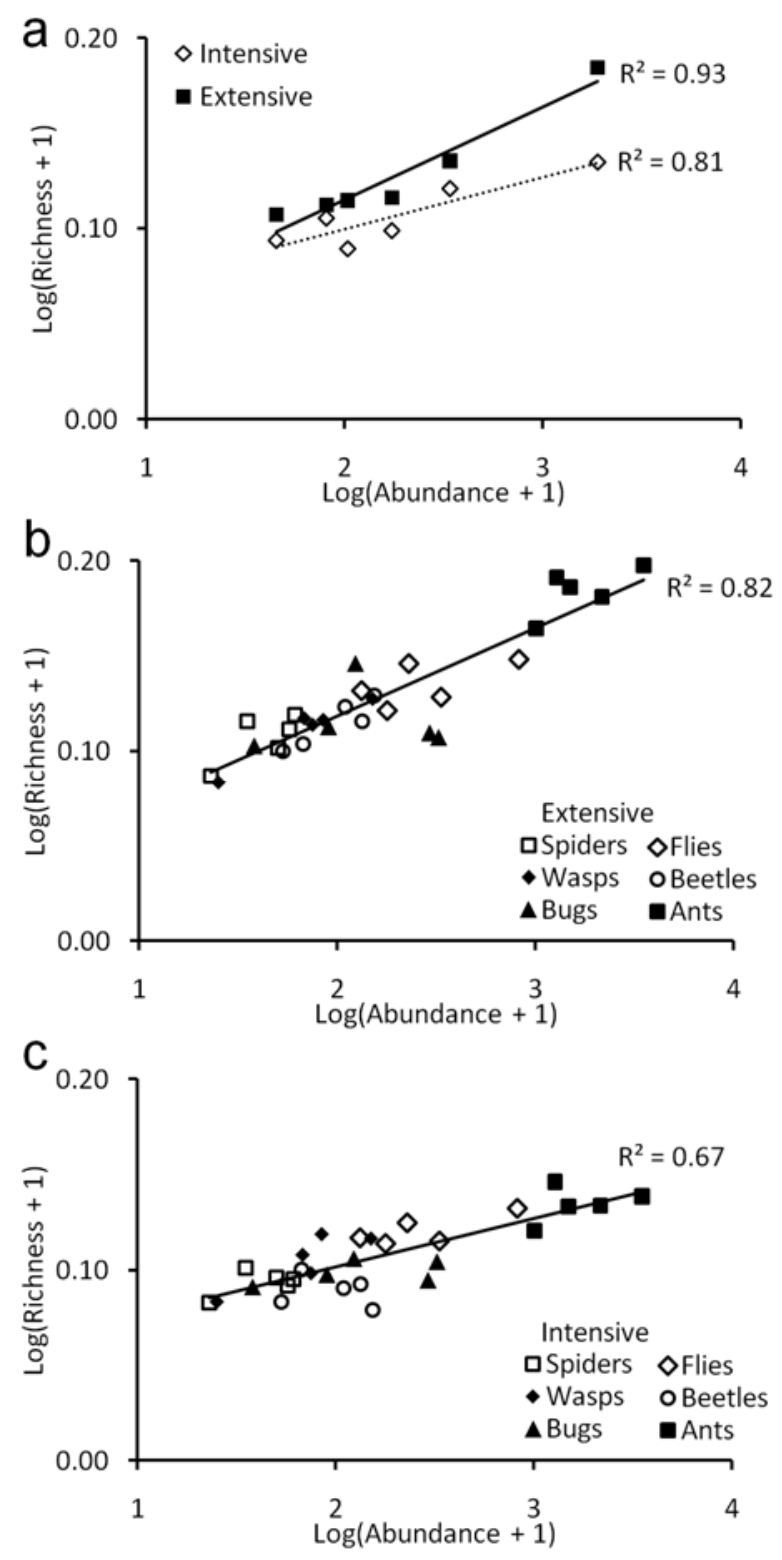

Fig. 5. The average proportion of landscape scale species richness captured by intensive and extensive sampling in relation to the abundance sampled from the landscape. Panel (a) has one sample for each taxon, and the abundance and richness have been averaged over the five landscapes. Panels (b) and (c) show the results for extensive and intensive sampling respectively, where each taxon has one point for each landscape. 


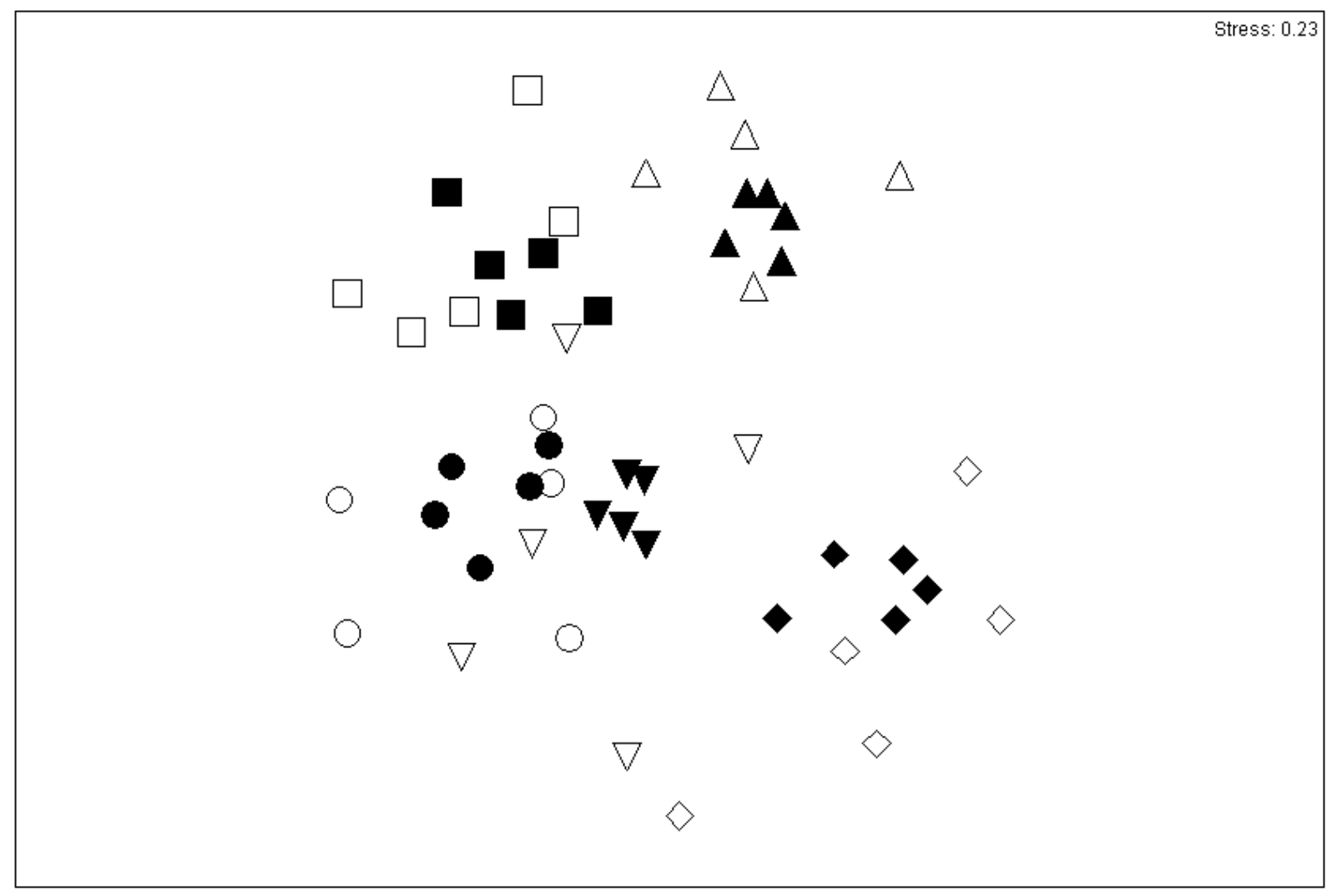

Fig. 6. A Multi-Dimensional Scaling (MDS) plot illustrating the similarity in species composition between intensive (hollow symbols) and extensive (solid symbols) samples. Results from the two coastal landscapes are shown using squares and triangles, the two inland landscapes are shown using circles and inverted triangles, and the high elevation landscape is shown using diamonds. 